\title{
Understanding Bacterial Antibiotic Resistance and Pathogenicity Through Investigating Bacterial Membrane Proteins
}

\author{
Kangmin Duan ${ }^{1,2,3} \cdot$ Ayush Kumar ${ }^{3,4}$
}

Published online: 14 February 2018

(c) Springer Science+Business Media, LLC, part of Springer Nature 2018

The discovery of antibiotics is one of the most important breakthroughs in medical history. Countless lives have been saved from devastating infectious diseases. Many previously impossible clinical procedures, from cancer chemotherapy and organ transplantation, to radical replacement of diseased joints and coronary bypass have been enabled by the use of antibiotics. The availability of antibiotics is rightfully credited as one of the most important reasons for the extended human lifespan over the last century. However, the spread of antibiotic-resistance in bacterial pathogens, namely the capability of bacteria to resist treatment by an antimicrobial drug, is posing an ominous threat to human health once again. There are increasing cases of infections caused by bacteria resistant to multiple or all available antibiotics, leaving no treatment options for these patients. In addition to the human suffering, the health-care cost for the treatment of antibiotic resistance has increased dramatically. By 2050 , the estimated global financial cost of the antimicrobial resistance will be US $\$ 100$ trillion if the crisis is not tackled (Piddock 2016). In order to deal with antibiotic resistance crisis, it is necessary to understand how bacterial pathogens become resistance namely the mechanisms of antibiotic resistance, and how bacterial pathogens cause disease in the host, i.e., pathogenicity.

Kangmin Duan

Kangmin.Duan@umanitoba.ca

Ayush Kumar

Ayush.Kumar@umnaitoba.ca

1 Department of Oral Biology \& Medical Microbiology and Infectious Diseases, Rady Faculty of Health Sciences, University of Manitoba, Winnipeg, Canada

2 College of Life Sciences, Northwest University, Xian, China

3 Manitoba Chemosensory Biology Group, University of Manitoba, Winnipeg, Canada

4 Department of Microbiology, University of Manitoba, Winnipeg, Canada
Like in any other organisms, bacterial cell membranes are in the forefront to sense the environmental conditions and initiate a signaling transduction that enable bacteria to survive in either favorable conditions or adversary conditions. Such membrane-based signaling enables bacteria to utilize available nutrients, evade the host immune system, and withstand stress conditions such as the presence of antibiotics. Hence, investigating the molecular mechanisms of membrane-related process provides a unique opportunity to understand antibiotic resistance and bacterial pathogenicity.

This special issue features eight excellent papers reporting some of the exciting new findings or trends regarding signaling and molecular mechanisms located on bacterial membranes. The topics cover the function and regulation of efflux pumps that are involved in antibiotic and biocide susceptibility (Slipski et al. 2017) and adaptability (De Silva and Kumar 2017), the role of two-component regulatory systems (TCS) in regulating virulence (Mima et al. 2017) and life style (Bhagirath et al. 2017) and nutrient sensing (Steiner et al. 2018) and as a target for antimicrobial development (Cardona et al. 2017), ABC transporters involved in iron transport (Gao et al. 2017), and membrane proteins involved in energy production as a target against mycobacterial infections (Bown et al. 2017).

In the paper entitled "Effect of Sodium Chloride on Surface-Associated Motility of Acinetobacter baumannii and the Role of AdeRS Two-Component System" by De Silva and Kumar (2017), new insights on the regulation and function of the efflux pump AdeABC of the resistance-nodulation-division family in Acinetobacter baumannii are reported. Expression of AdeABC is known to be controlled by the AdeRS two-component system in this pathogen; however, new experimental results indicate that AdeRS not only modulates A. baumannii's antibiotic susceptibility, but also plays a role in biofilm formation as well as surface-associated motility. AdeRS deletion mutant shows more sensitive to saline stress. The results demonstrate that AdeRS could be part of adaptation strategy 
to salinity stress in A. baumannii, connecting antibiotic resistance and salinity stress.

The report "Biocide Selective TolC-Independent Efflux Pumps in Enterobacteriaceae" by Slipski et al. (2017) provides an excellent review of the efflux pumps involved in biocide resistance in Enterobacteriaceae. Bacterial resistance to biocides used as antiseptics and disinfectants is a growing concern in food preparation, agricultural, consumer manufacturing, and healthcare industries, particularly among Gram-negative pathogens in Enterobacteriaceae. Multidrug resistant efflux pumps represent an important biocide resistance mechanism in Enterobacteriaceae. The review provides an overview on the growing number of single component TolC-independent multidrug-resistant efflux pumps that are specifically associated with biocide resistance. It summarizes the known biocide substrates of these efflux pumps, compares their structural relatedness, Enterobacteriaceae distribution, and significance. Knowledge gaps are highlighted regarding the efflux-mediated resistome.

In "CmpX Affects Virulence in Pseudomonas aeruginosa Through the Gac/Rsm Signaling Pathway and by Modulating c-di-GMP Levels" Bhagirath et al. (2017) describe the role of a conserved cytoplasmic membrane protein $\mathrm{cmpX}$ (PA1775) in Pseudomonas aeruginosa. CmpX has been identified as a regulator of PA1611, a member of the central PA1611-RetS-GacS/A-RsmA/Y/Z pathway that controls several key virulence factors and biofilm formation in $P$. aeruginosa and hence the switch between chronic and acute infection status. New evidence is presented which demonstrates that $\mathrm{cmpX}$ regulates virulence, and controls biofilm formation in P. aeruginosa through PA1611-RetSGacS/A-RsmA/Y/Z, and possibly by modulating intracellular c-di-GMP levels. A cmpX knockout showed significantly decreased promoter activity of exoS an effector of the type three secretion system and increased activity of small RNA, RsmY. Importantly, cmpX mutant had elevated intracellular c-di-GMP level as well as increased expression of $w s p R$ (PA3702), a c-di-GMP synthase.

The paper entitled "Evidence of Cross-Regulation in Two Closely Related Pyruvate-Sensing Systems in Uropathogenic Escherichia coli" By Steiner et al. (2018) describes the interconnectivity between the metabolite-sensing TCSs YpdA/ YpdB and BtsS/BtsR in uropathogenic Escherichia coli (UPEC). The YpdA/YpdB and BtsS/BtsR TCSs are known to interact and alter the expression of putative transporter genes $y h j X$ and $y j i Y$ in $\mathrm{K} 12 \mathrm{E}$. coli. Evidence is presented to show that proper regulation of $y h j X$ in UPEC requires all components of these TCSs. Contrasting differences in the regulation of $y h j X$ and $y j i Y$ by these TCSs highlight that niche and lifestyle-specific pressures may be selecting for differential cross-regulation of TCSs in pathogenic and nonpathogenic E. coli.
The paper "Expression of Collagenase is Regulated by the VarS/VarA Two-Component Regulatory System in Vibrio alginolyticus" by Mima et al. (2017) identified the TCS VarS/VarA as the regulator of the gene colA encoding virulence factor collagenase in Vibrio alginolyticus. In vitro phosphorylation assays demonstrated that phosphorylated VarS acted as a phosphoryl group donor to VarA and the His300, Asp718 and His874 residues in VarS are essential for the phosphorylation whereas Asp54 residue in VarA is likely to receive the phosphoryl group from VarS. The results demonstrate that the VarS/VarA two-component regulatory system regulates the expression of collagenase in V. alginolyticus.

Another excellent review article in this special issue entitled "Essential Two-Component Systems Regulating Cell Envelope Functions: Opportunities for Novel Antibiotic Therapies" by Cardona et al. (2017) argues for that the bacterial signal transduction mechanisms, known as twocomponent systems (TCSs), are ideal antimicrobials targets. TCSs are unique in bacteria and control a diverse set of cellular functions including virulence, response to cell envelope stress, drug efflux and even viability. Because impaired TCSs affect antibiotic susceptibility in bacterial pathogens, TCSs potentially serves as targets of antibiotic adjuvant therapies. The review describes a list of essential TCSs, and their potentials as targets for antibacterial molecules. The methods for the identification of small molecules that inhibit TCSs and the challenges have been discussed.

In the paper "The Two-Operon-Coded ABC Transporter Complex FpvWXYZCDEF is required for Pseudomonas aeruginosa Growth and Virulence Under Iron-Limiting Conditions' by Gao et al. (2017) describes the genetic and functional roles of the newly identified transporter complex located on the envelop of $P$. aeruginosa. The data presented demonstrate that $f p v C D E F$ and $f p v W X Y Z$ (PA2403-2406) constituted an $\mathrm{ABC}$ transporter complex containing two operons: $f p v W X Y Z C D E$ and $f p v F$. Evidence is provided to show that the operon $f p v W X Y Z C D E$ is regulated by iron negatively while the single gene operon $f p v F$ is constitutively expressed. The results demonstrate that the $\mathrm{ABC}$ transporter complex encoded by $f p v W X Y Z C D E F$ plays important roles in growth, oxidative stress resistance, and virulence in $P$. aeruginosa.

In the review paper "Mycobacterial Membrane Proteins QcrB and AtpE: Roles in Energetics, Antibiotic Targets, and Associated Mechanisms of Resistance," Bown et al. (2017) provide an in-depth discussion on the discovery of mycobacterial QcrB and AtpE inhibitors, their modes of action, and the associated mechanisms of resistance observed to date. Since energy production using oxidative phosphorylation is essential for the survival of M. tuberculosis, even under conditions of dormancy, it presents an excellent target for anti-mycobacterial drugs. The inhibitors of 
QcrB and AtpE, which are part of the cytochrome $b c_{1}$ complex and $\mathrm{F}_{\mathrm{o}} \mathrm{F}_{1}$-ATP synthase, are among the most successful examples. These inhibitors are active against drug-resistant strains of M. tuberculosis, inhibit non-replicating cells, and also show potential for the treatment of other mycobacterial infections.

Understanding the mechanisms of antibiotic resistance and bacterial pathogenicity is essential in our combat against infectious diseases. The topics covered in this special issue on "Bacterial Membrane Proteins: Signal Transduction, antibiotic resistance and virulence" illustrate some exciting advances in our current understanding on how bacterial pathogen uses membrane-bound protein or protein complexes, such as efflux pumps, ABC transporters, and TCSs, to respond to environmental changes and to become resistant to antibiotics and biocide and cause disease.

\section{Reference}

Bhagirath AY, Somayajula D, Li Y, Duan K (2017) CmpX affects virulence in Pseudomonas aeruginosa through the Gac/Rsm signaling pathway and by modulating c-di-GMP Levels. J Membr Biol. https://doi.org/10.1007/s00232-017-9994-6

Bown L, Srivastava SK, Piercey BM, McIsaac CK, Tahlan K (2017) Mycobacterial membrane proteins QcrB and AtpE: roles in energetics, antibiotic targets, and associated mechanisms of resistance. J Membr Biol. https://doi.org/10.1007/s00232-017-9997-3

Cardona ST, Choy M, Hogan AM (2017) Essential two-component systems regulating cell envelope functions: opportunities for novel antibiotic therapies. J Membr Biol. https://doi.org/10.1007/s0023 2-017-9995-5

De Silva PM, Kumar A (2017) Effect of sodium chloride on surfaceassociated motility of Acinetobacter baumannii and the role of AdeRS two-component system. J Membr Biol. https://doi. org/10.1007/s00232-017-9985-7

Gao L, Guo Z, Wang Y, Wang Y, Wang K, Li B, Shen L (2017) The two-operon-coded ABC transporter complex FpvWXYZCDEF is required for Pseudomonas aeruginosa growth and virulence under iron-limiting conditions. J Membr Biol. https://doi.org/10.1007/ s00232-017-9979-5

Mima T, Gotoh K, Yamamoto Y et al (2017) Expression of collagenase is regulated by the VarS/VarA two-component regulatory system in Vibrio alginolyticus. J Membrane Biol. https://doi.org/10.1007/ s00232-017-9991-9

Piddock LJ (2016) Reflecting on the final report of the O'Neill review on antimicrobial resistance. Lancet Infect Dis 16(7):767-768

Slipski CJ, Zhanel GG, Bay DC (2017) Biocide selective TolC-independent efflux pumps in Enterobacteriaceae. J Membrane Biol. https://doi.org/10.1007/s00232-017-9992-8

Steiner BD, Eberly AR, Hurst MN, Zhang EW, Green HD, Behr S, Jung K, Hadjifrangiskou M (2018) Evidence of cross-regulation in two closely related pyruvate-sensing systems in uropathogenic Escherichia coli. J Membrane Biol. https://doi.org/10.1007/s0023 2-018-0014-2 